\title{
ENCEFALITIS DE BICKERSTAFF
}

\author{
BICKERSTAFF ENCEPHALITIS
}

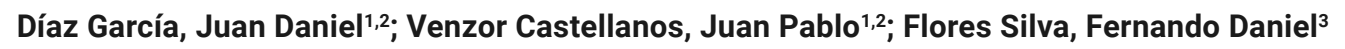

' Departamento de Medicina Interna, Hospital General "Dr. Manuel Gea González ", Ciudad de México, México.

2 Departamento de Neurología, Hospital General "Dr. Manuel Gea González ", Ciudad de México, México.

${ }^{3}$ Departamento de Neurología, Instituto Nacional de Ciencias Médicas y Nutrición "Salvador Zubirán", Ciudad de México, México.

Recibido: 16/10/2019 | Revisado: 02/11/2019 | Aceptado: 19/04/2020

DOI: 10.15568/am.2020.811.cc04

Actual Med. 2020; 105(817): 233-236

\section{Caso Clínico}

\section{RESUMEN}

Introducción: La encefalitis de Bickerstaff es una enfermedad neurológica aguda poco frecuente, que se caracteriza clínicamente por oftalmoplejía relativamente simétrica, ataxia, alteración del nivel de consciencia e hiperreflexia, perteneciendo a un espectro patológico continuo que incluye la oftalmoplejía aguda, síndrome de Miller Fisher y síndrome de Guillain-Barré.

Caso clínico: Femenino de 26 años de edad. Inició con visión borrosa, cefalea holocraneana, mialgias generalizadas y fiebre de $38.5^{\circ} \mathrm{C}$ de 7 días de evolución. Se encontró con amnesia, disartria, ataxia e hiperreflexia generalizada, paresia facio-braquio-crural izquierda y ptosis palpebral izquierda. Ante deterioro del nivel de consciencia progresivo se inició manejo avanzado de la vía aérea. Tomografía computarizada (TC) de cráneo con edema cerebral severo y resonancia magnética (RM) de cráneo con disminución de calibre de vasos corticales posteriores. Punción lumbar con disociación albumino citológica Electroencefalograma con encefalopatía con actividad de base tetha reactivo a maniobras de activación y potenciales evocados visuales con neuropatía óptica bilateral de tipo desmielinizante. Anticuerpos IgG antigángliosido con Anti-GQ1b positivos. Se inició tratamiento con inmunoglobulina a dosis de $0.4 \mathrm{~g} / \mathrm{kg} /$ día durante cinco días con posterior respuesta clínica satisfactoria.

Conclusión: El caso ilustrado reúne múltiples características de las descritas por Bickerstaff en sus series iniciales, como lo son la oftalmoplejía, la hiperreflexia y la alteración del estado de conciencia. La controversia en cuanto a si la encefalitis de Bickerstaff y el síndrome de Guillain-Barré son entidades diferentes, ya está planteada, así como la hipótesis alterna de que puede tratarse de enfermedades dentro de un mismo espectro.

\section{ABSTRACT}

Introduction: Bickerstaff encephalitis is a rare acute neurological disease, characterized clinically by relatively symmetric ophthalmoplegia, ataxia, altered level of consciousness and hyperreflexia, belonging to a continuous pathological spectrum that includes acute ophthalmoplegia, Miller Fisher syndrome and Guillain Barre syndrome.

Case report: Female, 26 years old. He started with blurred vision, holocranean headache, generalized myalgias and a $38.5^{\circ} \mathrm{C}$ fever of 7 days of evolution. He found amnesia, dysarthria, ataxia and generalized hyperreflexia, left facio-brachio-crural paresis and left palpebral ptosis. Faced with the deterioration of the level of progressive consciousness, advanced airway management began. Computed tomography (CT) of the skull with severe cerebral edema and magnetic resonance imaging (MRI) of the skull with reduced caliber of posterior cortical vessels. Lumbar puncture with cytological albumine dissociation Electroencephalogram with encephalopathy with tetha-based activity reactive to activation maneuvers and visual evoked potentials with bilateral optic neuropathy of demyelinating type. IgG antigangglioside antibodies with Anti-GQ1b positive. Immunoglobulin treatment was started at a dose of $0.4 \mathrm{~g} / \mathrm{kg} /$ day for five days with subsequent satisfactory clinical response. Conclussion: The illustrated case has multiple characteristics of those described by Bickerstaff in its initial series, such as ophthalmoplegia, hyperreflexia and altered state of consciousness. The controversy as to whether Bickerstaff's encephalitis and Guillain-Barré syndrome are different entities, is already raised, as well as the alternative hypothesis that it can be diseases within the same spectrum.

\author{
Palabras Clave: \\ Cuadriparesia; \\ Encefalitis de \\ Bickerstaff; \\ Hiperreflexia; \\ Oftalmoplejia; \\ Síndrome de \\ Guillain-Barre; \\ Síndrome de \\ Miller-Fisher.
}

Keywords:

Quadriparesis;

Bickerstaff Encephalitis;

Hyperreflexia;

Ophathamoplegia;

Guillain-Barre

Syndrome;

Miller-Fisher Syndrome. 


\section{INTRODUCCIÓN}

La encefalitis de Bickerstaff es una enfermedad neurológica rara que afecta el tronco encefálico, caracterizada por una constelación de signos y síntomas que incluyen oftalmoplejía aguda, ataxia y alteraciones en el estado de conciencia, así como signos piramidales (1-3).

El estudio de laboratorio que más apoya el diagnóstico es la presencia de autoanticuerpos anti GQ1b, especialmente tomando en cuenta el contexto clínico. Es importante recordar que la ausencia de los anticuerpos no descarta la enfermedad, además de que se pueden presentar en otras patologías (síndrome de Miller-Fisher, síndrome de Guillain-Barré) (4-8).

El tratamiento de la encefalitis de Bickerstaff, así como de las otras variantes del síndrome anti GQ1b, requiere el reconocimiento y posterior confirmación diagnóstica. Aunque los síntomas neurológicos iniciales pueden ser alarmantes, suele autolimitarse. La mayoría de los pacientes tiene un buen pronóstico, y alcanzan recuperación espontánea completa en un periodo promedio de 6 meses desde el diagnóstico (9-10).

\section{CASO CLÍNICO}

Femenino de 26 años, sin antecedentes crónico-degenerativos y con antecedentes ginecobstétricos: G1 A0 P0 C1. Inició con cuadro clínico de 7 días de evolución, caracterizado por visión borrosa, cefalea holocraneana con Escala Visual Análoga (EVA) del dolor 5/10 de inicio súbito, mialgias generalizadas, fiebre de $38.5^{\circ} \mathrm{C}$. A su ingreso hospitalario presentó los siguientes signos vitales: Tensión arterial 110/70 mmHg, Frecuencia cardíaca 90 latidos por minuto, Frecuencia respiratoria 20 respiraciones por minuto, Temperatura $38.3^{\circ} \mathrm{C}$. A la exploración física se encontró con amnesia de hechos recientes, disartria, ataxia a pequeños pasos, hiperreflexia generalizada de predominio en extremidades superiores, paresia facio-braquio-crural izquierda 4 sobre 5 , sensibilidad conservada y ptosis palpebral izquierda. El fondo de ojo, el electrocardiograma y la radiografía de tórax son normales. La analítica (hemograma, coagulación, proteína $\mathrm{C}$ reactiva, bioquímica, función tiroidea, uroanálisis urocultivo y hemocultivos) dentro de parámetros normales.

Posteriormente la paciente comienza con un deterioro del nivel de consciencia progresivo con depresión respiratoria, por lo que se inició manejo avanzado de la vía aérea.

Se realizó Tomografía computarizada (TC) de cráneo para abordaje de deterioro neurológico, en el que se observó la presencia de edema cerebral severo, sin evidencia de lesiones focales o difusas a nivel de parénquima. Resonancia magnética $(\mathrm{RM})$ de cráneo con disminución de calibre de vasos corticales posteriores. Se realizó manejo y medidas antiedema cerebral, posterior a la resolución de esta condición, se realizó punción lumbar en donde se reportó: aspecto claro; glucosa $79 \mathrm{mg} / \mathrm{dl} ; 5$ células/ml, hiperproteinorraquia $123 \mathrm{mg} / \mathrm{dl}$. Se solicitó perfil viral: Virus de Hepatitis B y C y VIH negativos, reacción en cadena de polimerasa (PCR) para virus y hongos, tinción de Ziehl Neelsen, VDRL y tinta china en líquido cefalorraquídeo (LCR) negativos. Se solicitó electroencefalograma en el que se reportó encefalopatía con actividad de base tetha reactivo a maniobras de activación y potenciales evocados visuales con neuropatía óptica bilateral de tipo desmielinizante. Ante la sospecha de una causa inmunológica de encefalitis no infecciosa, se solicitaron anticuerpos IgG antigángliosido con Anti-GQ1b positivos.

Ante un diagnóstico presuntivo de encefalitis de Bickerstaff, se inició tratamiento con inmunoglobulina a dosis de $0.4 \mathrm{~g} / \mathrm{kg} /$ día durante cinco días. Tres días posteriores ante una mejoría clínica importante la paciente es extubada.

Una semana después la paciente no presenta amnesia ni disartria, los reflejos osteotendinosos normales, con menor oftalmoplejía, siendo egresada con independencia completa para las actividades de la vida diaria.

\section{DISCUSIÓN Y CONCLUSIÓN}

En 1951 Bickerstaff y Cloake reportaron 3 casos de somnolencia, oftalmoplejía y ataxia, a los que diagnosticaron como una encefalitis circunscrita al mesencéfalo y al rombencéfalo (1). Propusieron como criterios para el diagnóstico: oftalmoplejía externa simétrica y progresiva y ataxia, en presencia de alteraciones del estado de consciencia o hiperreflexia (2).

Se le considera como una enfermedad dentro del espectro del síndrome de Guillain-Barre y el síndrome de Miller-Fisher. La presencia de anticuerpos anti-GQ1b es una característica en común, por lo que se les considera dentro del síndrome anti-GQ1b (3). La mayor parte de los pacientes tienen antecedente de una infección previo al inicio de los síntomas neurológicos (usualmente de tracto respiratorio superior), y siendo los microorganismos más frecuentemente implicados C. jejuni y H. Influenzae (4).

Por tanto, el principal diagnóstico diferencial por su similitud en cuanto a cuadro clínico es el síndrome de Miller-Fisher. En el caso de este último, se ve la triada clásica de ataxia, oftalmoplejía y arreflexia. Sin embargo, en el caso de que se encuentren asociada al- 
teraciones en el estado de conciencia o hiperreflexia, se prefiere el diagnóstico de encefalitis de Bickerstaff como reflejo de la participación del sistema nervioso central, específicamente del tronco encefálico (Tabla 1) (5). Por ende la principal diferencia en el diagnóstico clínico son las alteraciones en estado de consciencia, la cual es variable y puede ir desde pacientes que se presentan somnolencia, estupor o en casos más severos coma (se ve hasta en un $20 \%$ de los pacientes) (6).

El abordaje también se puede complementar con estudios de Resonancia Magnética (RMN) y electroencefalografía (EEG), ya que la afectación principal es de sistema nervioso central. Por EEG, se pueden observar ondas lentas dentro de rangos normales, consistentes con la implicación del SNC. Los hallazgos por RMN son variables, donde se pueden observar lesiones hipointensas en T1 e hiperintensas en T2, las cuales se observan sobre todo en tronco encefálico (7).

Revisiones publicadas recientemente permitirían diferenciar dos tipos de encefalitis de Bickerstaff. Por un lado, la forma típica, con características neurológicas y serológicas muy parecidas al síndrome de Miller Fisher y con rápida recuperación. Y, por otro lado, la forma atípica, con negatividad para los anticuerpos IgG anti-GQ1b, hallazgos en el líquido cefalorraquídeo atípicos, hallazgos en neuroimagen poco concluyentes y evolución más tórpida (8).

El tratamiento se fundamenta en corregir el trastorno autoinmune y para ello lo que ha mostrado mayor efectividad es la inmunoglobulina humana por vía intravenosa, habiéndose utilizado también inmunoadsorción con columnas conjugadas de triptófano, plasmaféresis, esteroides y diversas combinaciones de los anteriores (9).

El pronóstico suele ser bueno y normalmente a los sei meses en el $66 \%$ de los pacientes está en completa remisión, el $11 \%$ continúa con síntomas leves y un $23 \%$ presenta secuelas más importantes (déficits sensitivos y/o motores, diplopía permanente y ataxia), requiriendo un $16 \%$ durante su evolución ventilación mecánica y siendo la muerte un desenlace muy poco frecuente $(5 \%)(10)$.

En conclusión, el caso ilustrado reúne múltiples características de las descritas por Bickerstaff en sus series iniciales, como son la oftalmoplejía, la hiperreflexia y la alteración del estado de conciencia. La controversia en cuanto a si la encefalitis

Características requeridas para el diagnóstico

\section{Encefalitis de Bickerstaff}

Oftalmoplejía progresiva y relativamente simétrica con ataxia durante 4 semanas.

Alguna alteración de la consciencia (coma, semicoma o estupor) o signos piramidales (hiperreflexia o reflejos patológicos).

Fuerza de las extremidades de 5 o 4 puntos en la escala del Medical Research Council.

\section{Síndrome de Miller Fisher}

Oftalmoplejía progresiva y relativamente simétrica con ataxia durante 4 semanas

Hiporreflexia o arreflexia

Fuerza de las extremidades de 5 o 4 puntos en la escala del Medical Research Council

\section{Características de soporte para el diagnóstico}

Antecedentes de síntomas infecciosos en las 4 semanas previas al inicio de la clínica neurológica.

Disociación albúmino-citológica en el líquido cefalorraquídeo.

Presencia de anticuerpos IgG antigangliósidos GQ1b.

Tabla 1. Criterios diagnósticos para encefalitis de Bickerstaff y síndrome de Miller Fisher. 
de Bickerstaff y el síndrome de Guillain-Barré son entidades diferentes ya está planteada, así como la hipótesis alterna de que puede tratarse de enfermedades dentro de un mismo espectro. Así lo ilustra la similitud bioquímica de ambas, como sucede en nuestro caso, en el que se evidenció la presencia de anticuerpos GQ1b, situación que es común en el síndrome de Guillain-Barré. Sin embargo, los hallazgos clínicos no orientan a esta última, como la alteración del estado de conciencia, ya que la oftalmoplejía es igualmente definitoria del síndrome de Miller-Fisher, una variante del síndrome de Guillain-Barré.

Otro punto a tener en cuenta es la respuesta terapéutica. En las series de casos descritas desde las primeras publicaciones, se ha observado mejoría al iniciarse el tratamiento con dosis altas de esteroides parenterales, inmunoglobulina o plasmaféresis, sin diferencias significativas entre estas últimas. La evolución de este paciente a lo largo de la estancia hospitalaria, así como en los meses subsiguientes, también sugiere el diagnóstico de encefalitis de Bickerstaff.

Son necesarios más estudios para precisar las características moleculares y bioquímicas que permitan orientar y especificar el diagnóstico de encefalitis de Bickerstaff, con vistas a optimizar su tratamiento, y acelerar la recuperación y funcionalidad de los pacientes, más aún, dado que el proceso de diagnóstico diferencial redunda en un mayor consumo de tiempo y en el retraso de la mejoría de los pacientes.

\section{REFERENCIAS BIBLIOGRÁFICAS}

1. Odaka M. Bickerstaff's brainstem encephalitis: clinical features of 62 cases and a subgroup associated with Guillain-Barre Syndrome. Brain. 2003; 126:2279-90. DOI: 10.1093/brain/awg233

2. Horton E, Krishnamoorthy S, Reynolds L. Bickerstaff's encephalitis. BMJ Case Rep. 2014; 126:2279-90. DOI: 10.1136/bcr-2014-205336

3. Ito M. Bickerstaff's brainstem encephalitis and Fisher syndrome form a continuous spectrum: Clinical analysis of 581 cases. J Neurol 2008; 255:674-682. DOI: 10.1007/ s00415-008-0775-0

4. Shahrizaila N. Bickerstaff brainstem encephalitis and Fisher syndrome: anti-GQ1b antibody syndrome $J$ Neurol Neurosurg Psychiatry 2013; 84:576-583. DOI: 10.1136/ jnnp-2012-302824

5. Roos R. An Elderly Patient With Bickerstaff Brainstem Encephalitis and Transient Episodes of Brainstem Dysfunction. Arch Neurol 2008; 65:821-824 DOI: 10.1001/ archneur.65.6.821
6. Damasceno A, França M Jr, Pimenta D, De Deus-Silva L, Nucci A, Damasceno B. Bickerstaff encephalitis, Guillain-Barré syndrome and idiopathic intracranial hypertension: are they related conditions? Arq Neuropsiquiatr. 2008; 66:744-6. DOI: 10.1590/s0004$282 \times 2008000500027$

7. Overell JR, Willison HJ. Recent developments in MiIler- Fisher syndrome and related disorders. Curr Opin Neurol. 2005; 18:562-6. DOI: 10.1097/01. wco.0000173284.25581.2f

8. Yuki N. Fisher syndrome and Bickerstaff brainstem encephalitis. J Neuroimmunol. 2009; 215:1-9. DOI: 10.1016/j.jneuroim.2009.05.020

9. Yuki N, Shahrizaila N. How do we identify infectious agents that trigger Guillain-Barré syndrome, Fisher syndrome and Bickerstaff brainstem encephalitis. J Neurol Sci. 2011; 302:1-5. DOI: 10.1016/j.jns.2010.12.010

10. García, J, Calleja S, Fernández JM, Lahoz CH. Romboencefalitis de Bickerstaff. Neurología. 2005; 20:366-9.

\section{CONFLICTO DE INTERESES}

Los autores/as de este artículo declaran no tener ningún tipo de conflicto de intereses respecto a lo expuesto en el presente trabajo.

\section{Si desea citar nuestro artículo:}

Díaz García JD, Venzor Castellanos JP, Flores Silva FD. Encefalitis de Bickerstaff. Actual Med. 2020;811(105):233-236. DOI: 10.15568/am.2020.811.cc04 